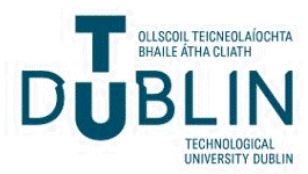

Technological University Dublin

ARROW@TU Dublin

\section{Concentric double hollow grid cathode discharges}

\author{
C. T. Teodorescu-Soare \\ Universitat Innsbruck \\ S. A. Irimiciuc \\ Alexandru loan Cuza University \\ C. Ionita \\ Universitat Innsbruck
}

See next page for additional authors

Follow this and additional works at: https://arrow.tudublin.ie/scschphyart

Part of the Physics Commons

\section{Recommended Citation}

C.T. Teodorescu-Soare, S.A. Irimiciuc, C. Ionita, D.G. Dimitriu, B. Hodoroaba, T. O'Hara, O. Vasilovici, L. Amarandi, R.W. Schrittwieser, Concentric double hollow grid cathode discharges, International Journal of Mass Spectrometry, Volume 436, 2019, Pages 83-90, ISSN 1387-3806, DOI: 10.1016/j.jjms.2018.11.019.

This Article is brought to you for free and open access by the School of Physics \& Clinical \& Optometric Science at ARROW@TU Dublin. It has been accepted for inclusion in Articles by an authorized administrator of ARROW@TU

Dublin. For more information, please contact

arrow.admin@tudublin.ie, aisling.coyne@tudublin.ie, gerard.connolly@tudublin.ie. Funder: CNCS-UEFISCDI; CEEPUS

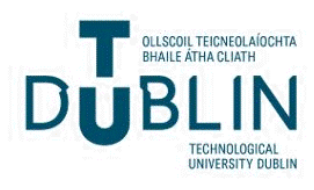




\section{Authors}

C. T. Teodorescu-Soare, S. A. Irimiciuc, C. Ionita, D. G. Dimitriu, B. Hodoroaba, T. O'Hara, O. Vasilovici, L. Amarandi, and R. W. Schrittwieser

This article is available at ARROW@TU Dublin: https://arrow.tudublin.ie/scschphyart/152 
Full Length Article

\title{
Concentric double hollow grid cathode discharges
}

\author{
C.T. Teodorescu-Soare ${ }^{\mathrm{a}, \mathrm{b}}$, S.A. Irimiciuc ${ }^{\mathrm{b}, \mathrm{c}}$, C. Ionita $^{\mathrm{a}}$, D.G. Dimitriu ${ }^{\mathrm{b}}$, B. Hodoroaba ${ }^{\mathrm{b}, \mathrm{d}}$, \\ T. O'Hara ${ }^{\mathrm{e}}$, O. Vasilovici ${ }^{\mathrm{a}}$, L. Amarandi ${ }^{\mathrm{b}}$, R.W. Schrittwieser ${ }^{\mathrm{a}, *}$
}

a Institute for Ion Physics and Applied Physics, University of Innsbruck, Technikerstr. 25, A-6020, Innsbruck, Austria

${ }^{\mathrm{b}}$ Faculty of Physics, Alexandru Ioan Cuza University, 11 Carol I Blvd., 700506, Iasi, Romania

${ }^{c}$ National Institute for Laser, Plasma and Radiation Physics - NILPRP, 409 Atomistilor Street, Bucharest, Romania

${ }^{\mathrm{d}}$ University of Bucharest, Faculty of Physics, Bucharest-Magurele, Romania

e Dublin Institute of Technology, Kevin Street, Dublin 2, D08 X622, Ireland

\section{A R T I C L E I N F O}

\section{Article history:}

Received 14 September 2018

Received in revised form 5 November 2018

Accepted 9 November 2018

Available online 17 November 2018

Dedicated to the 75th birthday of Helmut Schwarz, a great scientist and friend of the Innsbruck University Institute for Ion

Physics and Applied Physics for many years

\section{Keywords:}

Plasma physics

Electric discharges

Plasma discharge

Double layers

Spherical grid discharge

Double grid discharge

Cathode discharge

Inverted fireball

Multiple complex space charge structures

Plasma diagnostics

Langmuir probe

Plasma potential

Floating potential

Electron temperature

Electron density

Ion density

Non-linear dynamics

Current oscillations

\begin{abstract}
A B S T R A C T
A new cathode system, consisting of two concentric spherical hollow grids with two aligned orifices, is investigated by space-resolved Langmuir probe measurements and non-linear dynamics analysis. Negative biases of this spherical hollow grids arrangement lead to the formation of two complex space charge structures in the regions of the orifices. The overall dynamics of the current-voltage characteristic ( $I-V$ characteristic) of each discharge is characterized by strong oscillatory behaviour with various waveforms correlated with jumps in the static $I-V$ characteristics. Space-resolved measurements through the two aligned orifices of the two grids show a peak increase of the electron temperature and particle density in the regions of the two space-charge structures. The effects of the biases and Ar pressure on the overall spatial distribution of all plasma parameters are investigated. Two important working points of the concentric double hollow grid cathode discharges are revealed which could make this configuration suitable as an electron source.
\end{abstract}

(c) 2018 Elsevier B.V. All rights reserved.

\section{Introduction}

Multiple complex space-charge structures that occur in unmagnetized low temperature plasmas, such as fireballs or inverted fireballs, arise from ionization phenomena near electrodes or due to locally applied constraints [1-5]. The generation of such com-

\footnotetext{
* Corresponding author.

E-mail address: roman.schrittwieser@uibk.ac.at (R.W. Schrittwieser).
}

plex space-charge structures, usually bounded by plasma double layers, is often accompanied by plasma instabilities such as complex and/or high-frequency oscillations and strongly non-linear behaviour [2,4,6-9]. In recent years strong emphasis has been laid on the dynamics of such individual structures generated on various geometrical electrode configurations [9-16], as well as on the interactions between multiple complex space-charge structures $[9,12,13]$. Theoretical models based on the fractal nature of plasma particle geodesics around these structures have also been implemented in order to understand their complex dynamics [9]. 


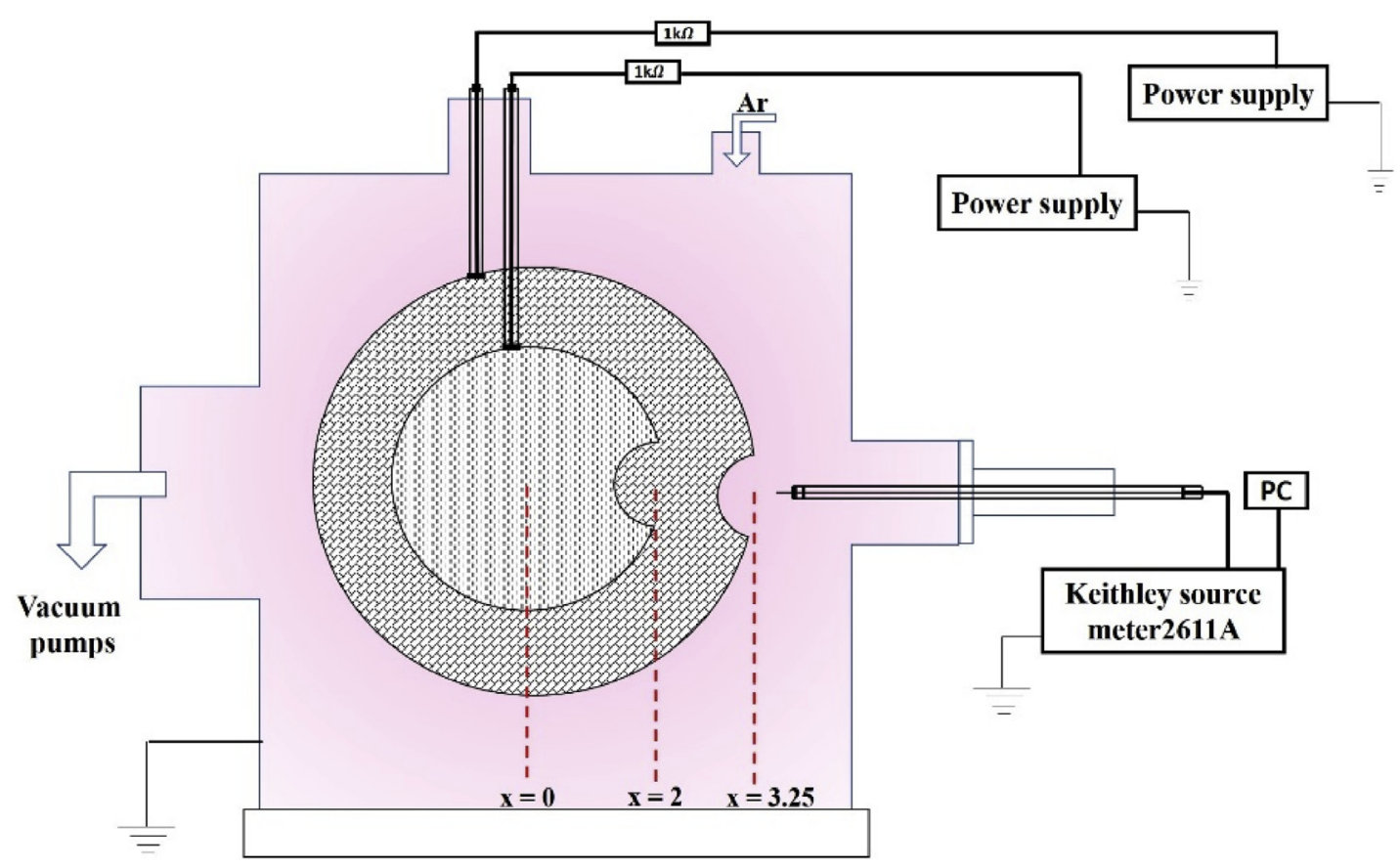

Fig. 1. Experimental arrangement of the Innsbruck set-up. The distance $x$ from the centre outward is measured in $\mathrm{cm}$.

Inverted fireballs got this name due to their appearance inside a usually spherical hollow grid electrode. They have recently been used for both fundamental investigations in various background gases $[6,14,15,17,18]$ and applications such as inertial electrostatic confinement fusion $[10,11]$, space propulsion systems [16], sputtering, thin film deposition and surface processing [19]. Due to the relative novelty of this discovery there are only few papers covering the dynamics of the complex phenomena involved in a discharge in such a configuration and thus the understanding of its applications and limits is rather scarce.

Our previous contributions focused on an alternative configuration consisting of a highly transparent spherical grid with a small orifice, where we discussed the appearance of a fireball in the outer region and a high ion density plasma inside the grid. The fireball appears as a quasi-spherical luminescent discharge through the orifice, having a clear boundary inside the grid, continuing outside with a diffuse electron beam which gradually fades $[12,13]$. Discharge configurations using spherical cathodes with an orifice are often used in applications of high temperature electron beams impinging on neutral plasmas like DC-electron bombardment ion thrusters [20]. There are, however, some limitations for a single grid configuration since electron temperature or densities strongly depend on the working conditions coupled with the need of subsequent acceleration of the electrons in order to attain the necessary conditions for specific applications. Thus, there is still need of research towards a fundamental understanding of the configuration consisting of a hollow spherical grid cathode with an orifice in order to optimize it and determine clear working regimes that can lead to the development of future applications.

In this paper we report on the investigation of a concentric double hollow grid cathode discharge using Langmuir probes and nonlinear dynamics methods. The Langmuir probe measurements are focused on determining the plasma parameters like electron temperature, plasma potential or particle density across the axis of the double grid system and presenting the effect of the bias applied on the outer grid. The effect of the pressure on the plasma parameters was also investigated in order to have a general overview of the plasma dynamics in this double-grid discharge. The global discharge current was evaluated in various bias configurations, presenting oscillatory regimes strongly dependent on the external parameters, in particular background pressure and discharge voltage. Different oscillation regimes are shown and investigated.

\section{Experimental set-up}

The core of the experimental arrangement consists of two concentric spherical stainless steel gridded cathodes with aligned orifices. The inner grid has $4 \mathrm{~cm}$ diameter, the outer grid $6.5 \mathrm{~cm}$. The grids have a mesh width of $0.25 \mathrm{~mm}$, the diameter of the grid wires is $0.1 \mathrm{~mm}$. The diameter of the orifice in the inner grid is $3 \mathrm{~mm}$, in the outer grid $5 \mathrm{~mm}$. During the experiments both the outer and inner grid were negatively biased with respect to the electric ground of the set-up.

Similarly as in our earlier investigations with a single spherical grid cathode [12,13], complementary experiments have been performed in two plasma experiments, one at the University of Innsbruck (the Ïnnsbruck set-up), and the other one at the University of Iaşi (the Ïaşi set-up), both described in detail in [12,13]. The main differences between the two devices are the material of the wall (stainless steel in the Innsbruck set-up, glass in the Iaşi set-up). In the cylindrical stainless steel chamber of the Innsbruck set-up the electric ground was the chamber itself. In the bell-shaped glass chamber experiment in the Iaşi set-up an additional circular stainless steel plate of $3 \mathrm{~cm}$ diameter served as grounded anode.

In spite of the different chambers the observed phenomena were similar in both devices. Especially there was no qualitative difference in the observations whether the inner or the outer grid voltage was varied while the other grid was kept on a constant negative voltage. In both chambers the working gas was argon. The gas flow was adjusted by electronic MKS mass flow controllers. The working pressure in each set-up was produced by a vacuum system consisting of a preliminary pump (Pfeiffer Vacuum DUO $10 \mathrm{M}$ ) and controlled by a pressure measuring system. Before each experiment the chambers were cleaned and the ambient gas was pumped out by a high vacuum turbo-molecular pump (Pfeiffer Vacuum TC600 PM C01 720) to a residual pressure of about $2 \times 10^{-5}$ mbar. 
In the Innsbruck set-up (see Fig. 1) the discharge was ignited by a constant voltage of $-350 \mathrm{~V}$ with respect to ground on the inner grid, while the outer grid bias was varied. We also investigated the influence of the argon pressure on the discharge by adjusting the gas flow in the chamber. The working pressure was varied between $7 \times 10^{-2}$ and $2.5 \times 10^{-1}$ mbar.

In the Innsbruck set-up an axially movable cylindrical tungsten Langmuir probe with $1 \mathrm{~mm}$ length and $0.125 \mathrm{~mm}$ diameter was used for measurements in axial direction through the aligned orifices of the two spherical grid cathodes from the centre $(x=0)$ up outward to $x=4.5 \mathrm{~cm}$ (see Fig. 1). A Keithley Instruments $2600 \mathrm{~A}$ System SourceMeter was used to record the current-voltage characteristic ( $I-V$ characteristic) of the probe with a spatial resolution of $1 \mathrm{~mm}$. Each characteristic was recorded with an integration time of $10 \mathrm{~s}$. Although more reliable for direct measurements of the plasma potential, emissive probes could unfortunately not be used since they have always led to strong perturbations and the creation of localized discharges around them.

In order to investigate the nonlinear dynamics of the complex plasma structures, in the glass-bell chamber of the Iaşi set-up time series of the discharge current oscillations and their corresponding FFTs were recorded during forward and backward sweeps of the applied voltage on the inner grid. In addition static currentvoltage characteristics were taken of the inner grid and evaluated with emphasis to current jumps, hysteresis and other related phenomena.

\section{Results and discussions}

\subsection{Static current-voltage characteristic (Iaşi set-up)}

Fig. 2 shows the static current-voltage characteristic of the inner discharge (i.e. between the inner grid and the anode), while keeping a constant voltage of $V_{\text {ext }}=-300 \mathrm{~V}$ on the outer grid and an argon pressure of $p=7 \times 10^{-2}$ mbar. The discharge shows two current jumps ( $A \rightarrow B$ and $C \rightarrow D$ in Fig. 2$)$ accompanied by hysteresis effects. After the first jump of the current, the inner discharge is ignited, as can be observed in Fig. 3a. After the second jump of the current, also the outer discharge is ignited (see Fig. 3b).

\subsection{Langmuir probe measurements (Innsbruck set-up)}

Langmuir probe investigations were performed in a wide range of background pressures and bias configurations. For all experiments discussed in the following the primary discharge is that one which is ignited at first between the inner grid and the walls of the chamber for a voltage of $V_{\text {int }}=-350 \mathrm{~V}$.

The secondary discharge, i.e. the one between the outer grid and the chamber wall is ignited in a wide range of voltages $(-11 \mathrm{~V}$ $\left.\geq V_{\text {ext }} \geq-350 \mathrm{~V}\right)$. The voltage applied on the outer grid $\left(V_{\text {ext }}\right)$ will further be considered as a control parameter to which we will refer throughout the following discussion. For each bias configuration the probe was moved from the space outside the grids ( $x$ $\geq 4.6 \mathrm{~cm}$ from the centre of the inner grid) towards the centre of the inner grid $(x=0 \mathrm{~cm})$. At each position the probe voltage was varied between $-70 \mathrm{~V}$ and $-15 \mathrm{~V}$, with the limits of the measurement constantly adapted in order to take into account the changes in the plasma potential and to avoid secondary emission from the probe and the possible formation of a plasma structure around it that might disturb the main discharges.

For small absolute values of the voltage on the outer grid $\left(V_{\text {ext }}=\right.$ $-11 \mathrm{~V}$, see Figs. $4 \mathrm{a}$ and $4 \mathrm{~b}$ ) the source of the discharge is the inner grid. A dumbbell structure is formed at the entrance of the inner grid (similar as in Fig. 3a), while the overall discharge presents similar features as in the case of a single grid $[12,13]$. Relatively far away

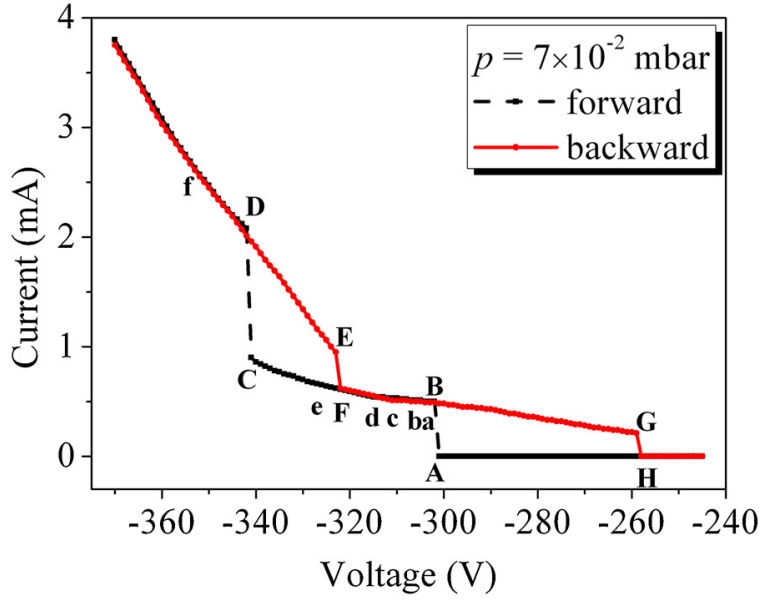

Fig. 2. Static current-voltage characteristic of the inner discharge, recorded while the outer discharge voltage was $V_{\text {ext }}=-300 \mathrm{~V}$. The upper case letters correspond to the current jumps, while the lower case letters correspond to the positions on the characteristic where the oscillations of the discharge currents were recorded (see Fig. 8).

from the centre of the inner grid $(x \geq 4.6 \mathrm{~cm})$, all plasma parameters (electron temperature $T_{e}$, plasma potential $\Phi_{p l}$, floating potential $V_{f l}$, ion density $n_{i}$ and electron density $n_{e}$ ) are relatively constant (Fig. 4a and b). In the vicinity of the outer grid we notice exponential increases of $T_{e}$ from $0.3 \mathrm{eV}$ to $2.8 \mathrm{eV}$ and of $n_{e}$ from $10^{16} \mathrm{~m}^{-3}$ to $1.5 \times 10^{17} \mathrm{~m}^{-3}$. On the other hand, $\Phi_{p l}$ suffers only a slight increase from $-20 \mathrm{~V}$ to $-16 \mathrm{~V}$ and $V_{f l}$ has a quasi linear decrease from $-20 \mathrm{~V}$ to $-32 \mathrm{~V}$. In the proximity of the inner grid $T_{e}, n_{e}$ and $n_{i}$ reach their maxima, followed by a slower quasi-exponential decrease. It is noticeable, however, that the ion density reveals a significant increase starting from about $1 \mathrm{~cm}$ from the centre of the grid and reaching its maximum of about $3 \times 10^{14} \mathrm{~m}^{-3}$ around the centre of the inner grid for $x \cong 0$. Inside the inner grid, the plasma potential presents a drop of approximately $16 \mathrm{~V}$ (from $-16 \mathrm{~V}$ to $-32 \mathrm{~V}$ ), indicating a double layer formation in this region. These results are in line with our previous results presented in [13] where we found a strong ionization inside the inner grid and maxima of electron temperature and particle density in the vicinity of the spherical grid's orifice.

With the decrease of the voltage on the outer grid $\left(V_{\text {ext }}=-350 \mathrm{~V}\right.$, Fig. $4 \mathrm{c}$ and $\mathrm{d}$ ) the plasma becomes more intense and a second dumbbell structure appears in the outer grid's orifice range. The effect of the voltage on the overall values of the plasma parameters will be addressed later in this subsection. For equal voltages on both grids, the discharge becomes more luminous and the two dumbbell structures become well defined, easily distinguishable from the rest of the discharge (similar as in Fig. 3b). In terms of plasma parameters, the constant plateau is visible for $T_{e}$ and $\Phi_{p l}$ in the same region (from $x=4.6 \mathrm{~cm}$ up to $3.5 \mathrm{~cm}$ ) while the other parameters are showing exponential increases $\left(n_{i}\right.$ and $\left.n_{e}\right)$. The axial distribution reaches maxima for the previously mentioned parameters between the two grids followed by a slight decrease in temperature of about $3.8 \mathrm{eV}$ and a strong decrease in particle density (of about one order of magnitude for both electrons and ions). In the proximity of the inner grid's orifice the electron temperature, as well as electron and ion densities start to increase again, with the temperature reaching the same maxima as in the previous region, whilst the densities only show a slight increase from $2 \times 10^{18} \mathrm{~m}^{-3}$ to $6 \times 10^{18} \mathrm{~m}^{-3}$ for the electron density and $1.4 \times 10^{15} \mathrm{~m}^{-3}$ to $5 \times 10^{15} \mathrm{~m}^{-3}$ for the ion density. Inside the inner grid the electron temperature decreases and there is a steep increase in the particle density as it was the case for small values of $V_{e x t}$. Both $\Phi_{p l}$ and $V_{f l}$ present a decrease in the outer region of the electrodes and a drop in the proximity of the 


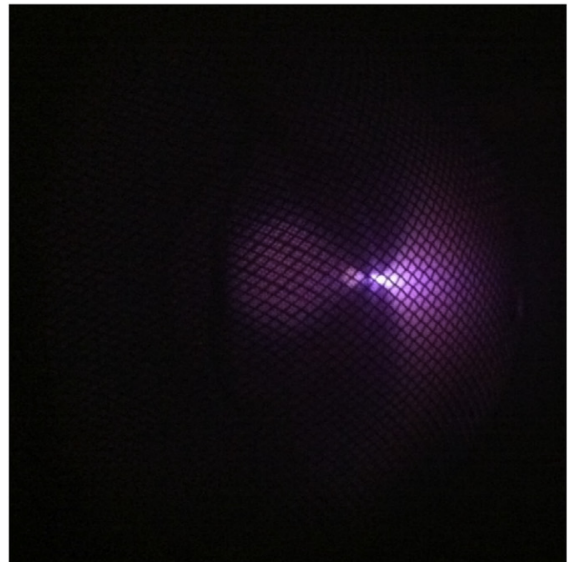

(a)

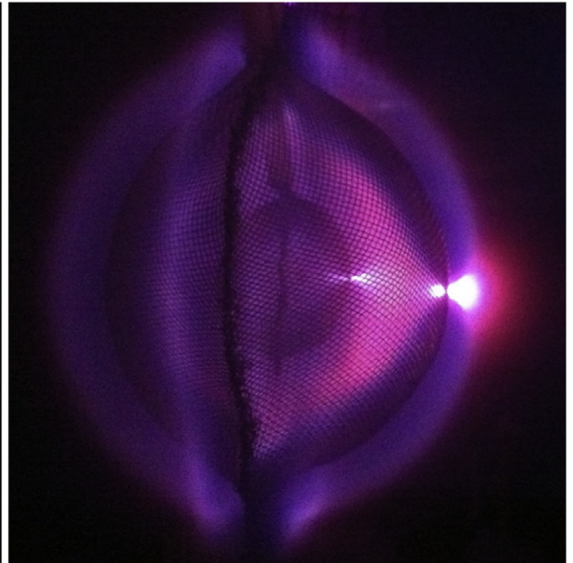

(b)

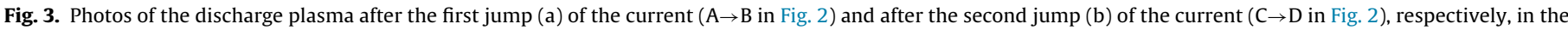
static current-voltage characteristic of the inner discharge.
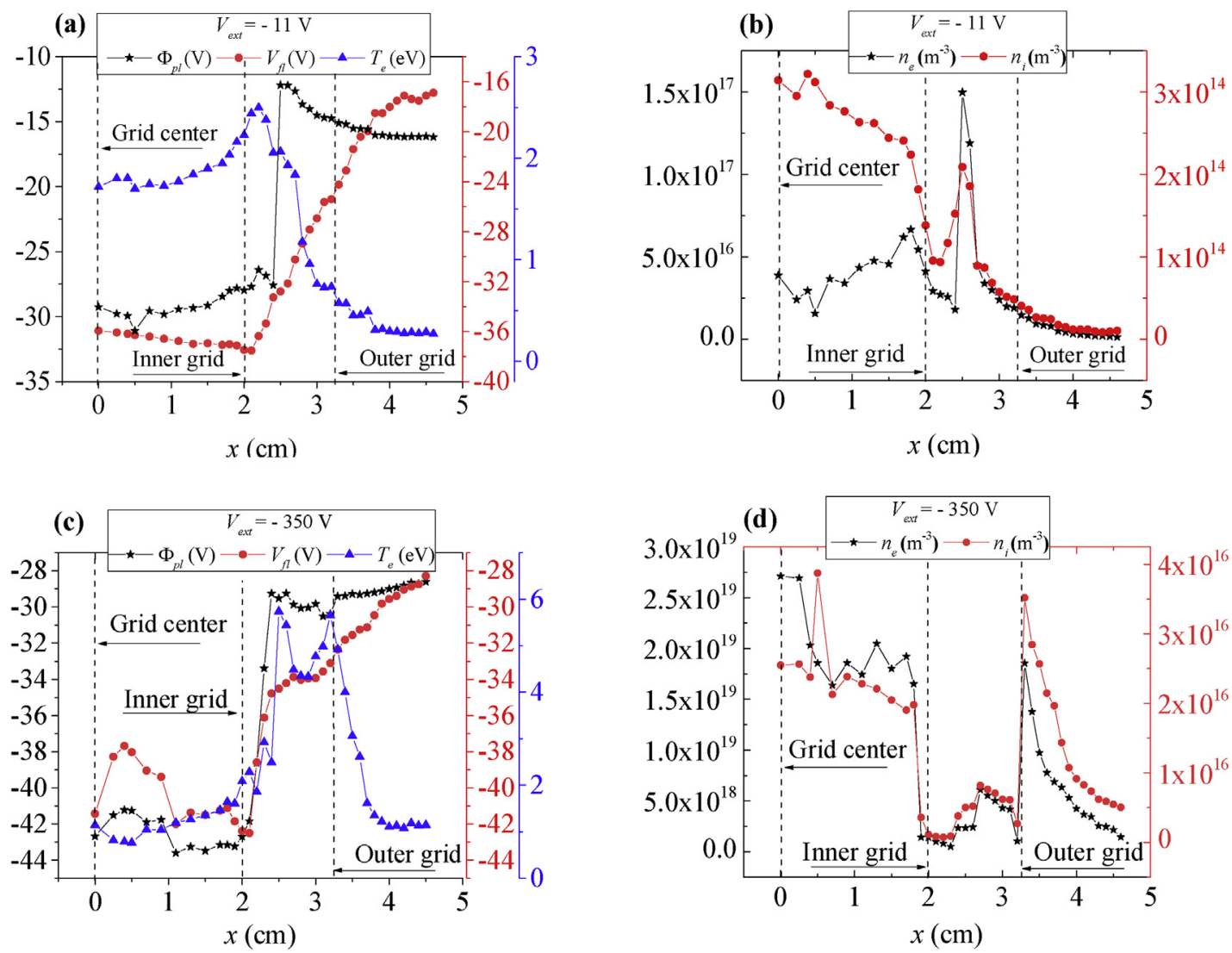

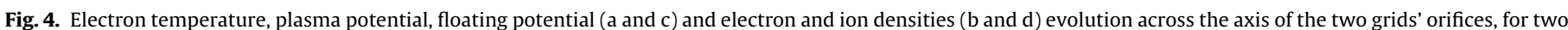

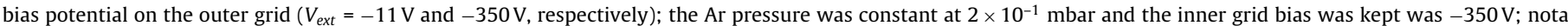

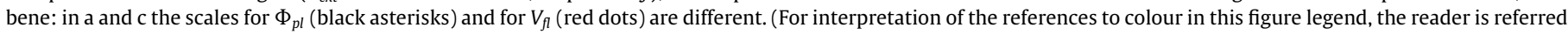
to the web version of this article).

outer grid ( $2 \mathrm{~V}$ for $\Phi_{p l}$ and $4 \mathrm{~V}$ for $V_{f l}$ ) indicating the formation of a secondary dumbbell structure which was visible with the naked eye. The presence of the dumbbell structure on the inner grid is indicated again by a more significant drop of about $14 \mathrm{~V}$ for $\Phi_{p l}$ and about $9 \mathrm{~V}$ for $V_{f l}$.

The Langmuir probe measurements have shown the importance of three main regions of the discharge that best represent the changes induced by the outer grid bias: the orifice of the outer grid $(x=3.25 \mathrm{~cm})$, the orifice of the inner grid $(x=2 \mathrm{~cm})$ and the interior of the inner grid $(0<x<1 \mathrm{~cm})$. If we present the previously discussed plasma parameters as functions of the outer grid bias (Fig. 5), we observe the presence of two different regimes:

The first regime, in a bias range of $-11 \mathrm{~V} \geq V_{\text {ext }} \geq-174 \mathrm{~V}$, is described by an enhancement in plasma temperature (Fig. 5a) and a steep decrease of both plasma potential and floating potential (Figs. 5b and 5c). In this regime the ion and electron densities show a slight increase (Fig. 5d and 5e - insert). We would like to note here that throughout the investigated plasma volume these depen- 
(a)

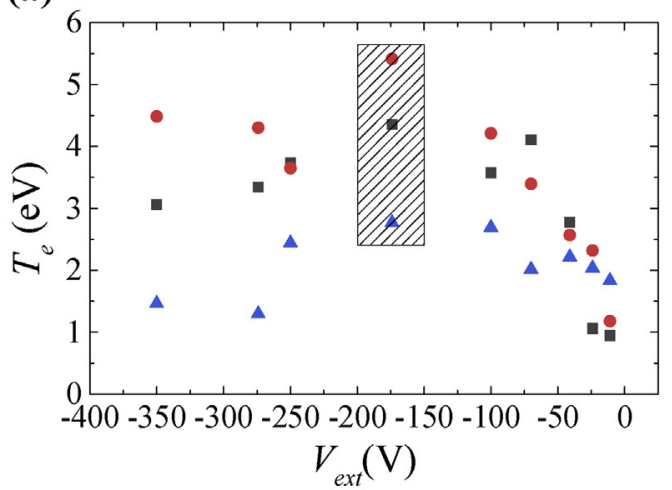

(c)

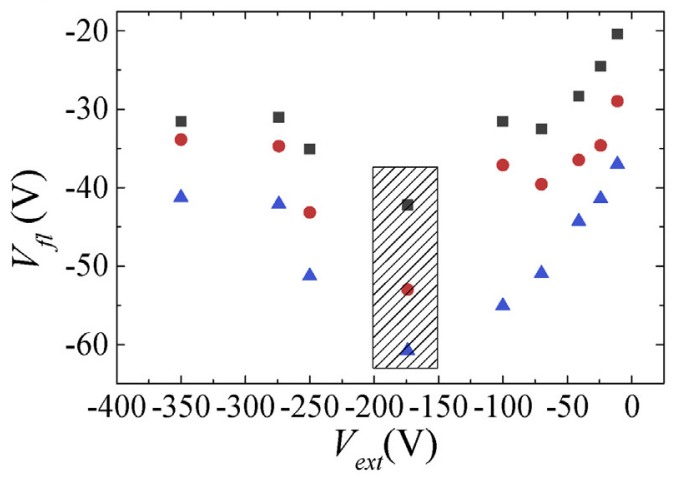

(b)

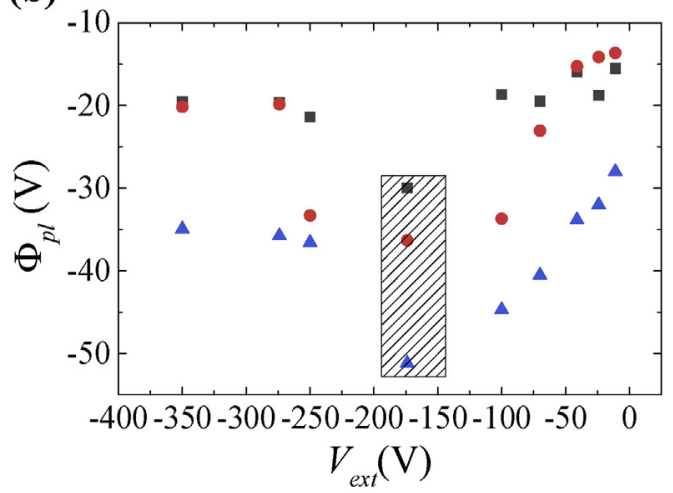

(d)

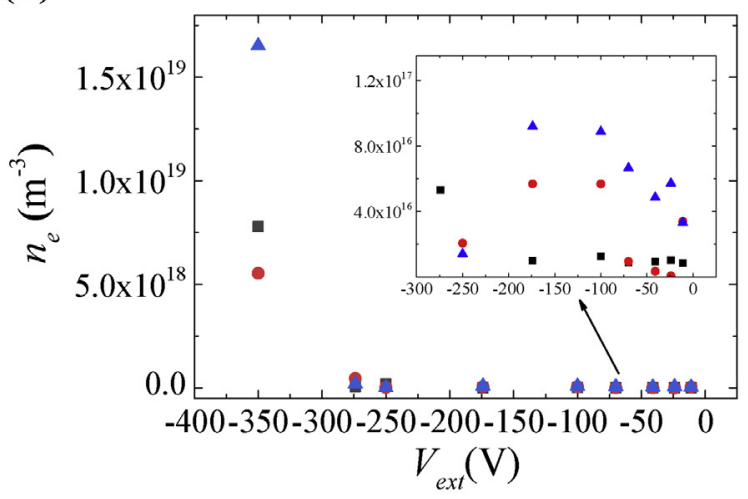

(e)

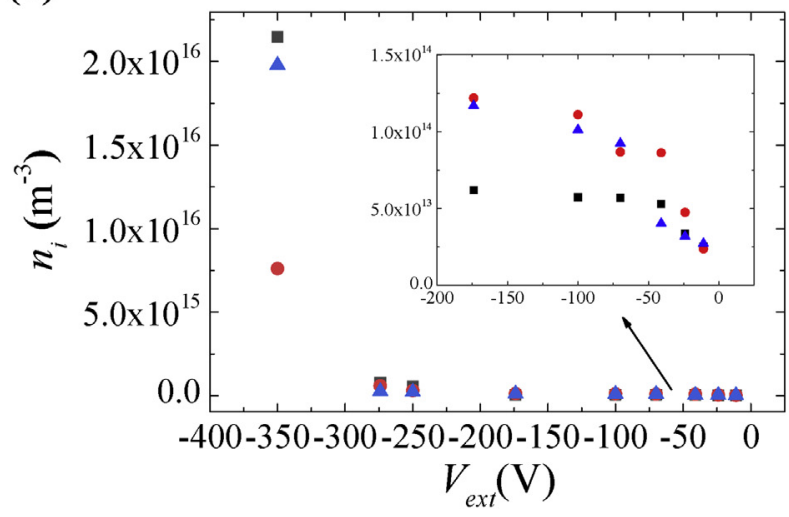

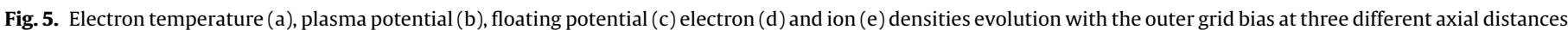

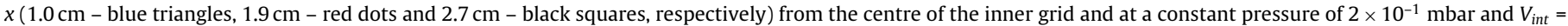

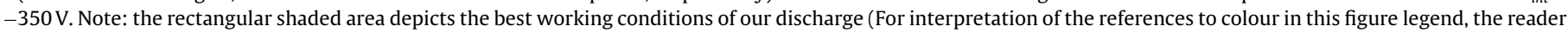
is referred to the web version of this article).

dences are maintained. A second region is noticed in the bias range $-174 \mathrm{~V} \geq V_{\text {ext }} \geq-350 \mathrm{~V}$, where an increase of $\Phi_{p l}$ and $V_{f l}$ of about $15 \mathrm{~V}$ is observed (Fig. $5 \mathrm{~b}$ and $\mathrm{c}$ ), as well as a decrease in the electron temperature of a few eV (Fig. 5a). In this regime we observe an exponential increase of both electron and ion densities reaching a maximum of $1.8 \times 10^{19} \mathrm{~m}^{-3}$ for $n_{e}$ and $2 \times 10^{16} \mathrm{~m}^{-3}$ for $n_{i}$ (Fig. $5 \mathrm{~d}$ and e). This comparative representation reveals the presence of an optimum for our discharge where we can generate relatively high electron and ion densities (around $10^{19} \mathrm{~m}^{-3}$ for electrons and around $10^{16} \mathrm{~m}^{-3}$ for ions) and high electron temperature $(5.5 \mathrm{eV})$, which could be a working regime for an electron source.
The complex space charge structures (the two plasma dumbbells) are three dimensional objects and the axial measurements provided a great deal of information regarding the distribution of energy and particles density along these structures. We have further attempted to modify their geometry by changing the working pressure and keeping the bias on the two grids constant $\left(V_{\text {ext }}=V_{\text {int }}\right.$ $=-350 \mathrm{~V}$ ) and see how this affects the overall distribution of the plasma parameters. The results are presented in Fig. 6, were the axial evolution of all the plasma parameters investigated until now is shown. We notice similar patterns as it was the case for $2 \times 10^{-1}$ mbar, presented above. There are two drops in plasma potential and floating potential, better seen here in the representation of 
(a)

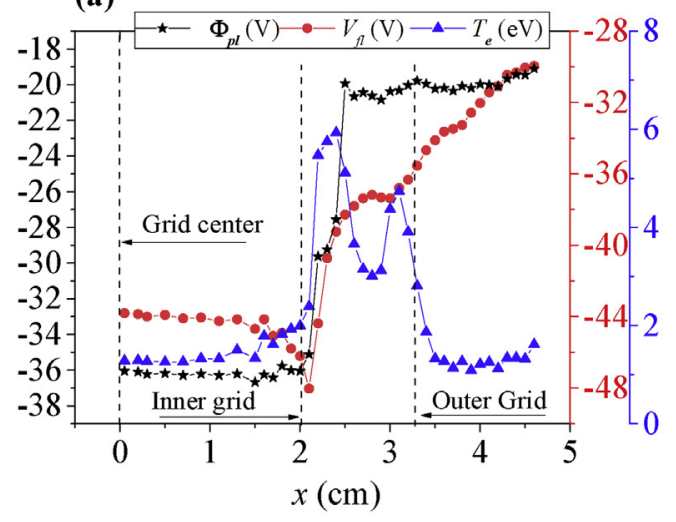

(b)

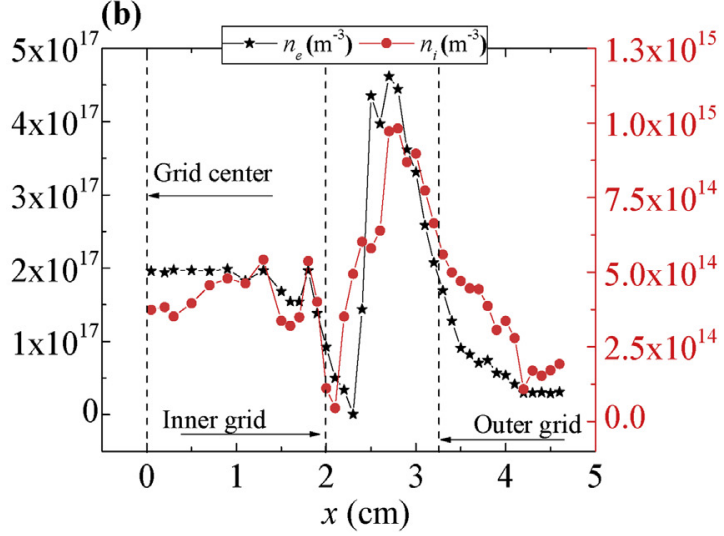

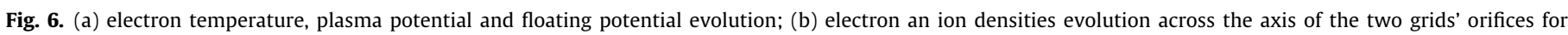
$p=9 \times 10^{-2}$ mbar and $V_{e x t}=V_{\text {int }}=-350 \mathrm{~V}$.

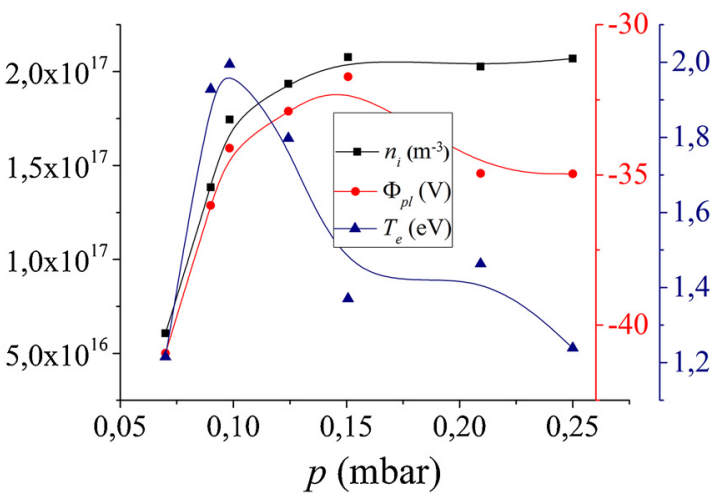

Fig. 7. Electron temperature, plasma potential and ion density versus background pressure $p$ at the axial position $x=1.9 \mathrm{~cm}$ from the centre of the inner grid.

the floating potential (Fig. 6a), while the electron temperature shows two maxima in the proximity of the orifices of each grid (Fig. 6a). There is, however, an expected decrease in particle densities (Fig. 6b) although the axial traces follow briefly the same evolution as in the previous case (Fig. 4e).

The overall effect of the background pressure $p$ is shown in Fig. 7 , where we have represented electron temperature, plasma potential and electron densities versus the background pressure only for one single axial position. Recognizing that the effect of the external parameters on the two plasma dumbbells is reflected throughout the volume of the discharge, and the evolution of $\Phi_{p l}$ is also reflected by $V_{f l}$ and $n_{i}$ and $n_{e}$, we only choose to represent $\Phi_{p l}$ and $n_{i}$. We notice that, with increasing pressure, the electron temperature increases until the pressure reaches $p=10^{-1} \mathrm{mbar}$, followed by a decrease probably induced by the increase of collisions with the background gas particles. The dependence of the plasma potential on the Ar pressure is described by a logarithmic function which increases with the pressure below $p=10^{-1}$ mbar, followed by a plateau. The ion density shows an increase followed by a saturation with the limit between the two regimes being the same pressure of $p=10^{-1}$ mbar. We highlight here again the possibility to generate high temperature electrons only for a small range of pressure (approximately $p=10^{-1}$ mbar), with reasonable electron and ion densities.

Taking into account the values of the plasma parameters from Figs. 4-7, we made an estimate of the ion and electron plasma frequencies, as well as the electron-neutral, electron-ion and ionneutral collision frequencies, respectively [21,22]. The ion plasma frequency is of the order of $\mathrm{MHz}$, comparable with the frequencies in Fig. 8 (see Section 3.3.), the electron plasma frequency is of the order of $\mathrm{GHz}$, well over the ranges of Fig. 8, while the electronneutral, electron-ion and ion-neutral collision frequencies have low values, well under the frequency ranges of Fig. 8. The critical value of the ion density for which there is a transition from a regime dominated by the electron-neutral collisions to a regime dominated by the electron-ion collisions was estimated as approximate $10^{13} \mathrm{~m}^{-3}$. This means that our discharge mostly works in the regime dominated by the electron-ion collisions.

\subsection{Non-linear dynamics analysis (Iaşi set-up)}

Information on the nonlinear dynamics of the discharges can be obtained by recording the oscillations of each of the two discharge currents. This has been done by using a digital oscilloscope, with a sampling rate of $2.5 \mathrm{GS} / \mathrm{s}$. Fig. 8 shows the inner discharge current oscillations and their corresponding Fast Fourier Transforms (FFTs). The oscillations of the outer discharge current were also recorded, but, for a given value of the voltage, the oscillations of the two discharge currents are similar, with small differences in the shape and amplitude, so these oscillations were not shown. After the first jump of the inner discharge current $(A \rightarrow B$ in Fig. 2, section 3.1.), double-period oscillations are found (Fig. 8a). Until the next jump of the current $(C \rightarrow D$ in Fig. 2$)$, the plasma system passes through different dynamic states, including intermittent mixed-modes oscillations with multiple time scales (Fig. 8b) [23], regular oscillations (Fig. 8c), intermittent oscillations (Fig. 8d) or noisy oscillations (Fig. 8e). After the ignition of the outer discharge, simultaneously with the second jump of the current in the static current-voltage characteristic ( $\mathrm{C} \rightarrow \mathrm{D}$ in Fig. 2), interesting reinforced damped oscillations can be observed (Fig. 8f). Moreover, for this case, the oscillations of the two discharge currents are in phase opposition, as can be observed in details in Fig. 9. This phenomenon deserves to be further investigated, in order to identify the oscillations' reinforcing mechanism. While decreasing the inner discharge voltage, the plasma system passes through almost the same dynamic states as described above, but of course in reverse order.

\section{Conclusion}

A concentric double hollow grid cathode discharge plasma was investigated by Langmuir probes, while its dynamics was investigated by recording and analysing the current-voltage characteristic of the inner cathode and the oscillations of the discharge current.

The static current-voltage characteristic of the inner discharge shows two jumps of the current accompanied by hysteresis effect, associated with the ignition of inner and outer discharge, respec- 

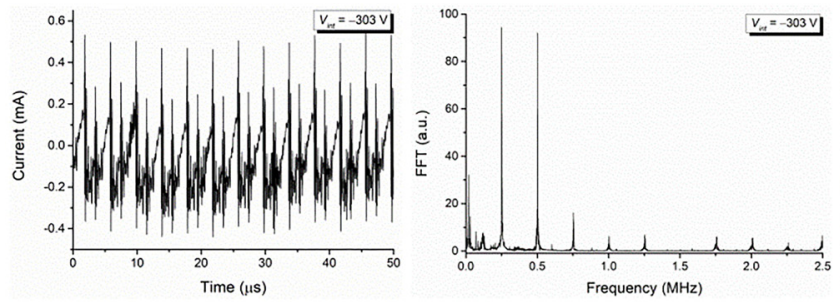

(a)
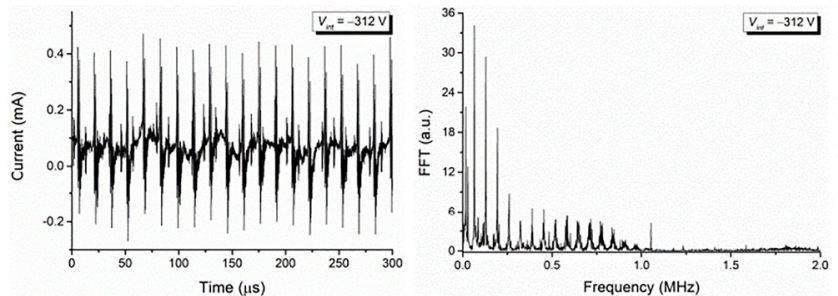

(c)
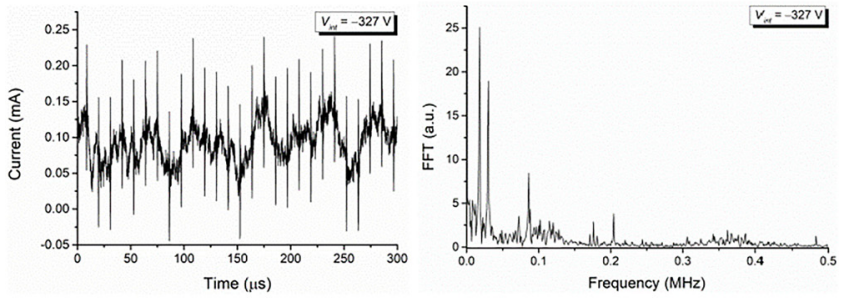

(e)
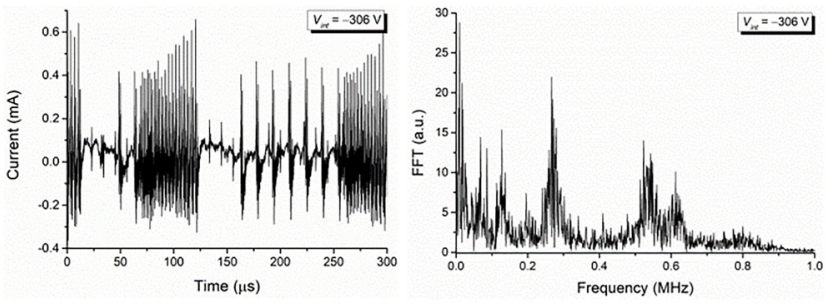

(b)
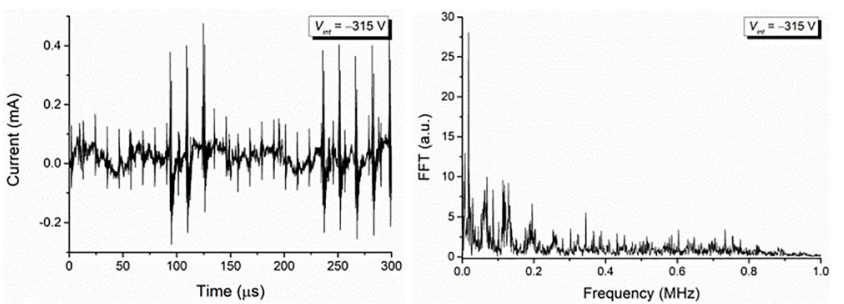

(d)
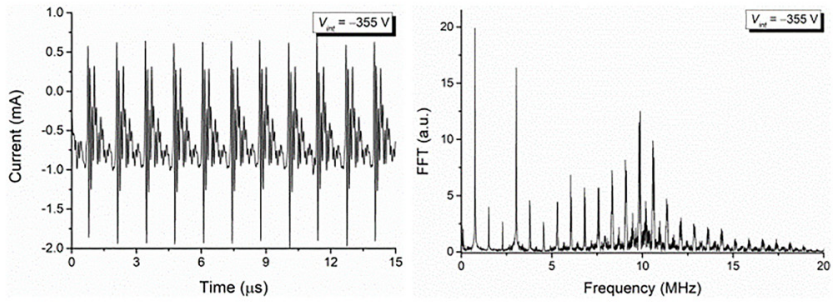

(f)

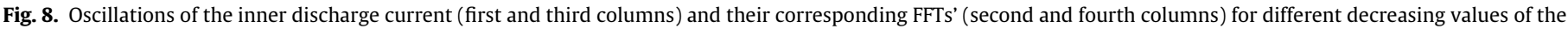
voltage applied on the inner grid.

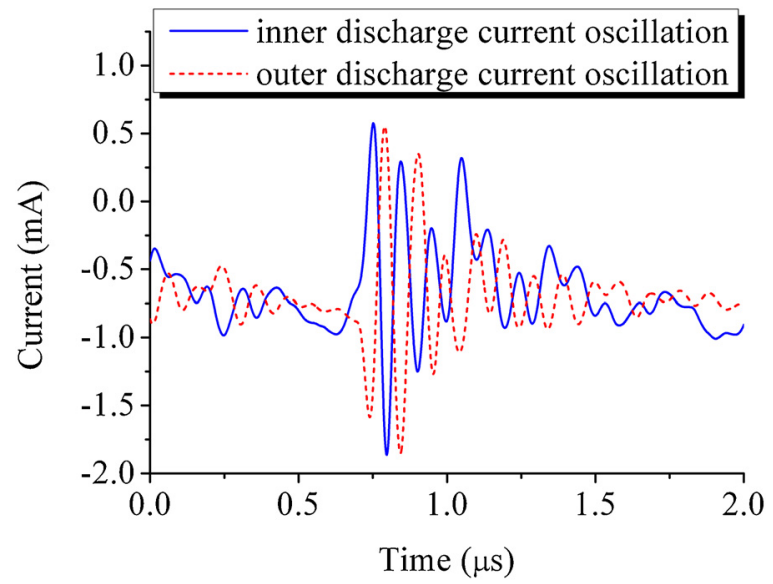

Fig. 9. Details of the inner and outer discharge currents oscillations, respectively, emphasizing their phase opposition.

tively. The recorded oscillations of the discharge current revealed that, by changing the voltage applied to the inner discharge, the plasma system passes through different complex dynamic states: intermittent mixed-modes oscillations with multiple time scales, intermittent oscillations and reinforced damped oscillations.

Probe measurements through the orifices provided the axial distribution of plasma and floating potentials, electron temperature, and electron and ion densities. Optimal conditions regarding pressure and voltages were established, in which such a discharge can function as an electron plasma source. Study of the background pressure influence on the discharge emphasized the existence of a threshold value of the pressure, up to which the values of all the plasma parameters change their increasing trend.

\section{Acknowledgments}

This work was supported by a grant of Ministry of Research and Innovation, CNCS-UEFISCDI, project number PN-III-P4-ID-PCE2016-0355, within PNCDI III, and by the CEEPUS network AT-0063.

\section{References}

[1] C. Ionita, D.G. Dimitriu, R.W. Schrittwieser, Elementary processes at the origin of the generation and dynamics of multiple double layers in DP machine plasma, Int. J. Mass Spectrom. 233 (2004) 343-354, http://dx.doi.org/10.1016/ j.ijms.2004.01.009.

[2] R.L. Stenzel, C. Ionita, R. Schrittwieser, Dynamics of fireballs, Plasma Sources Sci. Technol. 17 (035006) (2008) 11, http://dx.doi.org/10.1088/0963-0252/17/ 3/035006).

[3] N. Hershkowitz, Sheaths: more complicated than you think, Phys. Plasmas 12 (2005) 11, http://dx.doi.org/10.1063/1.1887189, 055502.

[4] D.G. Dimitriu, M. Aflori, L.M. Ivan, V. Radu, E. Poll, M. Agop, Experimental and theoretical investigations of plasma multiple double layers and their evolution to chaos, Plasma Sources Sci. Technol. 22 (2013), http://dx.doi.org/ 10.1088/0963-0252/22/3/035007), 035007.

[5] S. Gurlui, O. Niculescu, D.G. Dimitriu, C. Ionita, R.W. Schrittwieser, Elementary processes in the dynamics of two simultaneously excited fireballs in plasma, Int. J. Mass Spectrom. 365-366 (2014) 42-47, http://dx.doi.org/10.1016/j.ijms. 2014.01.002).

[6] R.L. Stenzel, J. Gruenwald, B. Fonda, C. Ionita, R. Schrittwieser, Trransit time instabilities in an inverted fireball 1. Basic properties; Phys. Plasmas 18 (2011) 012104 (9pp) (doi: https://doi.org/10.1063/1.3533437) and T̈ransit time instabilities in an inverted fireball 2. Mode jumping and nonlinearities; Phys Plasmas 18 (2011) 012104 (9pp) (doi: https://doi.org/10.1063/1.3533440).

[7] R.L. Stenzel, J. Gruenwald, C. Ionita, R. Schrittwieser, Pulsating fireballs with high frequency sheath-plasma instabilities, Plasma Sources Sci. Technol. 20 (2011) 9, http://dx.doi.org/10.1088/0963-0252/20/4/045017), 045017. 
[8] R.L. Stenzel, J.M. Urrutia, Öscillating plasma bubbles. I. Basic properties and instabilities; Phys. Plasmas 19 (2012) 082105 (10pp)

(doi.org/10.1063/1.4743019); Öscillating plasma bubbles. II. Pulsed experiments; Phys. Plasmas 19 (2012) 082106 (10pp)

(doi.org/10.1063/1.4743020); Öscillating plasma bubbles. III. Internal electron sources and sinks; Phys. Plasmas 19 (2012) 082107 (10pp)

(doi.org/10.1063/1.4743021); and Öscillating plasma bubbles. IV. Grids, geometry, and gradients; Phys. Plasmas 19 (2012) 082108 (10pp) (doi.org/10.1063/1.4743022).

[9] D.G. Dimitriu, S.A. Irimiciuc, S. Popescu, M. Agop, C. Ionita, R.W. Schrittwieser, On the interaction between two fireballs in low-temperature plasma, Phys.

Plasmas 22 (2015) 15, http://dx.doi.org/10.1063/1.4936106), 113511.

[10] R. Bandara, J. Khachan, Spherical ion oscillations in a positive polarity gridded inertial-electrostatic confinement device, Phys. Plasmas 20 (2013), http://dx. doi.org/10.1063/1.4813801, 072705.

[11] G.H. Miley, S. Krupakar Murali, Inertial Electrostatic Confinement (IEC) Fusion. Fundamentals and Applications, Springer, New York, 2014.

[12] C.T. Teodorescu-Soare, D.G. Dimitriu, C. Ionita, R.W. Schrittwieser, Experimental investigations of the nonlinear dynamics of a complex space-charge configuration inside and around a grid cathode with hole, Phys. Scr. 91 (2016) 7, http://dx.doi.org/10.1088/0031-8949/91/3/034002, 034002.

[13] R.W. Schrittwieser, C. Ionita, C.T. Teodorescu-Soare, O. Vasilovici, S. Gurlui, S.A. Irimiciuc, D.G. Dimitriu, Spectral and electrical diagnosis of complex space-charge structures excited by a spherical grid cathode with orifice, Phys. Scr. 92 (2017) 6, http://dx.doi.org/10.1088/1402-4896/aa5edd, 044001.

[14] D. Levko, Unmagnetized fireballs in the hollow cathode geometry, Phys. Plasmas 24 (2017) 6, http://dx.doi.org/10.1063/1.4983323, 053514.
[15] D. Levko, J. Gruenwald, On the energy deposition into the plasma for an inverted fireball geometry, Phys. Plasmas 24 (2017) 7, http://dx.doi.org/10. 1063/1.4998548, 103519.

[16] C. Syring, G. Herdrich, Jet extraction modes of inertial electrostatic confinement devices for electric propulsion applications, Vacuum 136 (2017) 177-183, http://dx.doi.org/10.1016/j.vacuum.2016.10.018.

[17] J. Gruenwald, J. Reynvaan, P. Knoll, Creation and characterization of inverted fireballs in $\mathrm{H}_{2}$ plasma, Phys. Scripta T161 (2014) 3, http://dx.doi.org/10.1088/ 0031-8949/2014/T161/014006), 014006.

[18] J. Gruenwald, J. Reynvaan, P. Geistlinger, Basic plasma parameters and physical properties of inverted He fireballs, Plasma Sources Sci. Technol. 27 (2018), http://dx.doi.org/10.1088/1361-6595/aaa332, 015008.

[19] P. Knoll, J. Reynvaan, M. Mayer, S. Surnev, P. Geistlinger, K. Krenn, K. Ettinger, C. Prietl, A. Hohenau, J. Gruenwald, B. Kunert, R. Resel, PECVD of carbon by inverted fireballs: from sputtering, bias enhanced nucleation to deposition, Diam. Relat. Mater. 65 (2016) 96-104, http://dx.doi.org/10.1016/j.diamond. 2016.02.021.

[20] C. Charles, Plasmas for spacecraft propulsion, J. Phys. D Appl. Phys. 42 (2009) 18, http://dx.doi.org/10.1088/0022-3727/42/16/163001), 163001.

[21] F.F. Chen, J.P. Chang, Lecture Notes on Principles of Plasma Processing, Plenum/Kluwer, New York, 2002

[22] A. Bers, Plasma Physics, Fusion Plasma, Electrodynamics, Oxford University Press, Oxford, 2016.

[23] P.K. Shaw, A.N. Sekar lyengar, Md. Nurujjaman, C̈anard and mixed mode oscillations in an excitable glow discharge plasma in the presence of inhomogeneous magnetic field, Phys. Plasmas 22 (2015), http://dx.doi.org/10. 1063/1.4936800, 122031. 